\title{
Sonochemically Fabricated AuPs/HRP/PANI Microelectrode Arrays
}

\author{
Lerdluck Kaewvimol, Stuart Collyer ${ }^{1}$, Seamus Higson ${ }^{1}$, \\ Chanchana Thanachayanont ${ }^{2}$ and Seeroong Prichanont ${ }^{*}$ \\ Department of Chemical Engineering, Faculty of Engineering, Chulalongkorn University, \\ Phayathai Rd., Bangkok 10330, Thailand \\ ${ }^{1}$ Cranfield Health, Cranfield University, Cranfield MK43 0AL, UK \\ ${ }^{2}$ National Metal and Materials Technology Center, Thailand Science Park, \\ Phaholyothin Rd., Phatumthani 12120, Thailand
}

(Received March 10, 2011; accepted May 31, 2011)

Key words: sonochemical fabrication, microelectrode arrays, enzyme biosensor, gold particles, horseradish peroxidase

This article is the first report on the sonochemical fabrication of enzyme/ gold particle microelectrode arrays. The study was aimed at incorporating the exceptional characteristics of both microelectrode arrays and gold particles (AuPs) for AuPs/horseradish peroxidase (HRP)/polyaniline (PANI) microelectrode array fabrication. A suitable sonication time of $17.30 \mathrm{~min}$ for microelectrode formation on a polydiaminobenzene (PDB)-modified glassy carbon electrode (GCE) was determined. The microelectrodes obtained were $\mu \mathrm{m}$ - and submicron-scale structures with population densities of approximately $7 \times 10^{4}$ and $17 \times 10^{4}$ pores $\mathrm{cm}^{-2}$, respectively. HRP/PANI microelectrode arrays were investigated in comparison to AuPs/HRP/PANI microelectrode arrays in terms of surface characteristics and electrochemical evaluation for phenol detection. Spherical AuPs of around $1 \mu \mathrm{m}$ were obtained by electrodeposition of AuP ions onto microelectrode surfaces. AuPs incorporated in the HRP/PANI matrix were postulated to enhance interfacial areas for HRP adsorption as well as function as electron conducting pathways between the redox HRP and electrode surfaces. As a consequence, a significantly improved 2.83-times-higher response current was obtained for the AuP-incorporated matrix in comparison to the ones without AuPs. Furthermore, the sensor response time of less than $20 \mathrm{~s}$ was achieved both with and without the incorporation of AuPs. Sonochemically fabricated enzyme/AuP microelectrode arrays showed good potential for biosensor applications.

"Corresponding author: e-mail: seeroong.p@chula.ac.th 


\section{Introduction}

Microelectrodes are small electrodes, which are conventionally assumed to have dimensions of $10 \mu \mathrm{m}$ or less, down to the submicron range. ${ }^{(1)}$ Microelectrodes of various geometries have been prepared mechanically or lithographically. In addition to single electrodes, microelectrode arrays have been prepared and used. These electrodes have shown good potential over the last two decades for diverse applications including electrochemical analysis and sensor technology.(1) This is because microelectrodes offer fast response time, elimination of mass convection effects on the fluctuation of current responses, and lowered detection limits ${ }^{(2,3)}$ in comparison with conventional planar electrodes. Microelectrode arrays can be fabricated by several approaches including photolithography, laser ablation, and recently, a sonochemical method, which is considered to be more cost effective for mass production. ${ }^{(3)}$ The construction of microelectrode arrays by sonochemical ablation has been investigated mainly on screenprinted electrodes for the detection of various compounds such as glucose using glucose oxidase, ${ }^{(2)}$ ethanol using alcohol oxidase, ${ }^{(4)}$ and pesticides using acetylcholinesterase $\mathrm{e}^{(5,6)}$ in which all of the enzymes are entrapped in polyaniline (PANI; a conducting polymer) matrices. Procedures for the production of microelectrode arrays by the sonochemical approach involve electrodeposition of a nonconductive polymeric film on electrode surfaces before sonochemical ablation of the polymer-modified electrode, resulting in microelectrode formation. An enzyme/conductive polymer could then be co-deposited on microelectrode surfaces for electrochemical analysis of various compounds. ${ }^{(2-6)}$ Recently, the emergence of nanoparticle technology has opened new eras for highly effective electrochemical assays, especially in biosensor applications. ${ }^{(1,7)}$ Gold particles (AuPs) have been extensively applied in the construction of biosensing devices owing to their extraordinary properties that allow the successful enhancement of biosensor

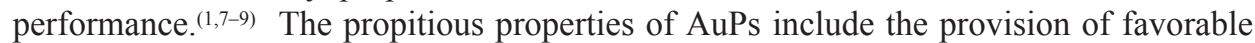
surfaces for stable enzyme immobilisation, allowance of direct electron transfer between a redox enzyme and the electrode surface without the need for electron mediators, provision of a high surface to volume ratio for enzyme immobilisation, and lessening of the enzyme-particles distance.(7) Thus, biosensors incorporated with AuPs have generally proved to have increased sensitivity, stability, and selectivity. ${ }^{(9,10)}$ Since AuPs can be easily assembled on glassy carbon electrodes, ${ }^{(1)}$ this work is therefore aimed at incorporating the exceptional characteristics of both microelectrode arrays and AuPs for the sonochemical fabrication of AuPs/horseradish peroxidase (HRP)/PANI microelectrode arrays, which is reported for the first time here. A suitable sonication time for microelectrode formation on a polydiaminobenzene-modified glassy carbon electrode was firstly determined. HRP/PANI microelectrode arrays were next investigated in comparison to AuPs/HRP/PANI microelectrode arrays in terms of surface characteristics and electrochemical evaluation for phenol detection. The results demonstrated significant electrochemical enhancement of the microelectrodes incorporated with AuPs, showing strong potential for biosensor applications. 


\section{Materials and Methods}

\subsection{Materials}

1,2 Diaminobenzene dihydrochloride, disodium hydrogen orthophosphate 12-hydrate, sodium dihydrogen orthophosphate 12-hydrate, sodium chloride, acetic acid, sodium acetate, and ferrocenecarboxilic acid (all analytical grade) were purchased from BDH (Poole, UK). Aniline hydrochloride, gold (III) hydrochloride trihydrate (ACS reagent; $\geq 49.0 \%$ AuPs basis), phenol, and horseradish peroxidase (HRP; E.C. 1.11.1.7; 167 purpurogallin units/mg; one purporogallin unit is defined as the production of $1.0 \mathrm{mg}$ purpurogallin from pyrogallol in $20 \mathrm{~s}$ at $\mathrm{pH} 6.0$ and $20^{\circ} \mathrm{C}$ ) were purchased from Sigma Aldrich (USA). All chemicals were used without further purification.

\subsection{Ultrathin film polymerisation}

A glassy carbon electrode (GCE) was first gently polished using 0.3 and $0.05 \mu \mathrm{m}$ alumina powders consecutively. The polished GCE was then cleaned in ethanol and distilled water consecutively in a bench top sonicated bath (Transonic T460, Germany) at $35 \mathrm{kHz}$ for 5 min each. A solution of $5 \mathrm{mM} \mathrm{1,2} \mathrm{diaminobenzene} \mathrm{dihydrochloride} \mathrm{was}$ prepared in phosphate buffer ( $\mathrm{pH} 7.4$ ) and was sonicated for 20 min for deoxygenation. The prepared solution was then electropolymerised on the GCE by potential sweep between 0 and $1,000 \mathrm{mV}$ versus $\mathrm{Ag} / \mathrm{AgCl}$ at $20 \mathrm{mVs}^{-1}$ for 50 cycles using $\mathrm{Pt}$ as a counterelectrode. All the electrodes were connected with a Sycopel Scientific AEW2 portable electrochemical workstation to a PC running ECProg3 software (Sycopel Scientific Ltd., Tyne and Wear, England). Then, the polydiaminobenzene (PDB; a nonconductive polymer)-coated electrode was dried for $2 \mathrm{~h}$ at room temperature and rinsed with deionised water for the removal of unpolymerised molecules.

\subsection{Sonochemical ablation of PDB film}

The modified GCE obtained in $\S 2.2$ was immersed into distilled water contained in a sonicated bath (Transonic T460, Germany) in the middle of the tank measured from all dimensions, and was clamped to a metal rod stand throughout the experiments. The effects of sonication time were studied by varying duration times at 16, 17.30, 18, and 19 min each at a fixed frequency of $35 \mathrm{kHz}$. Subsequently, the sonicated modified electrode was dried at room temperature for $1 \mathrm{~h}$, and cyclic voltammetry was performed in $5 \mathrm{mM}$ ferrocenecarboxilic acid (FCA) between -400 and $+800 \mathrm{mV}$ at $20 \mathrm{mVs}^{-1}$. Additionally, the amperometric responses were investigated at various FCA concentrations to test the performance of the modified electrode.

\subsection{Fabrication of HRP/PANI and AuPs/HRP/PANI microelectrode arrays}

For the fabrication of HRP/PANI microelectrodes, the sonochemically prepared microelectrodes (as in $\S 2.3$ ) were submerged in aniline/HRP acetate buffer solution (0.5 $\mathrm{mM}$ aniline, and $300 \mathrm{unit} / \mathrm{ml} \mathrm{HRP)} \mathrm{(} \mathrm{pH} 5.5$ ) for coelectropolymerisation by sequentially cycling the working electrode between -200 and $+800 \mathrm{mV}$ versus $\mathrm{Ag} / \mathrm{AgCl}$ for 20 cycles at $50 \mathrm{mVs}^{-1}$. Immediately following polymerisation, the working electrode was submerged in $\mathrm{pH} 7.4$ phosphate buffer to prevent enzyme denaturation and stored 
at $4{ }^{\circ} \mathrm{C}$ prior to use. The fabrication of $\mathrm{HRP} / \mathrm{AuPs} / \mathrm{PANI}$ microelectrode arrays was carried out similarly to the preparation of HRP/PANI microelectrodes; however, the electrodeposition of AuPs was achieved prior to HRP/PANI co-deposition. A solution of $0.3 \mathrm{mM}$ gold (III) hydrochloride trihydrate in distilled water was electrodeposited onto the sonochemically fabricated microelectrodes (as in $\S 2.3$ ) at $-20 \mathrm{mV}$ for $30 \mathrm{~s}$, which resulted in AuP deposition onto microelectrode surfaces. HRP/PANI co-deposition was finally achieved by the method described above. The microelectrodes obtained were model tested for amperometric responses (at a fixed potential of $-50 \mathrm{mV}$ and a sweep rate of $20 \mathrm{mVs}^{-1}$ ) to phenol at a concentration of $1 \times 10^{-6} \mathrm{M}$.

\subsection{Microelectrode surface characterisation}

Surface characterisation of all types of fabricated microelectrode arrays (PDB ablated, HRP/PANI on PDB ablated, AuPs on PDB ablated, and AuPs/HRP/PANI on PDB ablated) was performed by scanning electron microscopy (SEM; Joel JSM-6480LV, Japan).

\section{Results and Discussion}

The fabrication of HRP/PANI microelectrode arrays (Fig. 1(c)) or AuPs/HRP/PANI microelectrode arrays (Fig. 1(d)) could be achieved in different processing steps, as schematically shown in Fig. 1. A GCE was firstly modified by electropolymerisation

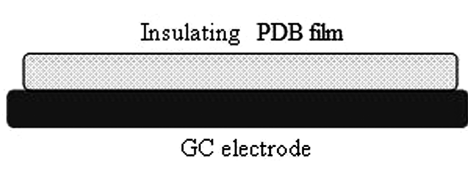

(a)

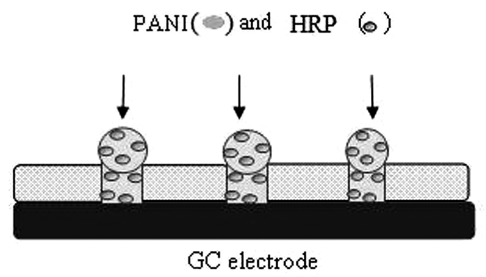

(c)

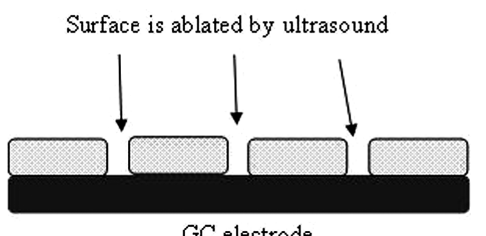

(b)

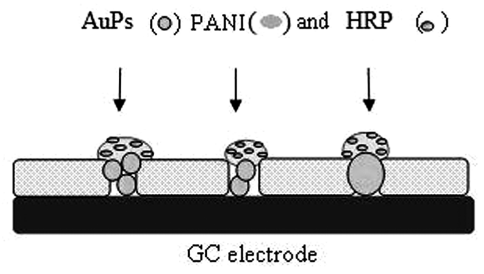

(d)

Fig. 1. Schematic diagrams of the planar glassy carbon electrode insulated via electrodeposition of polydiaminobenzene (a), sonochemical ablation of PDB film (b), HRP/PANI microelectrode arrays (c), and AuPs/HRP/PANI microelectrode arrays (d). 
of 1,2-diaminobenzene, forming an ultrathin nonconductive polymeric film of PDB on the glassy carbon surface (Fig. 1(a)). Microelectrode arrays were then achieved by sonochemical ablation at $35 \mathrm{kHz}$ for certain periods of time (Fig. 1(b)). Co-deposition of HRP/PANI could finally be achieved. For the case of AuPs incorporated on an electrode, gold ions were electrodeposited on the prepared microelectrode surfaces forming AuPs before the co-deposition of HRP/PANI, which finally resulted in AuPs/HRP/PANI microelectrode arrays.

\subsection{Formation of ultrathin PDB film on GCE}

A PDB-coated GCE was achieved by electropolymerisation of $5 \mathrm{mM}$ 1,2-diaminobenzene in phosphate buffer at pH 7.4 on the GCE. The observed CV is shown in Fig. 2(a). As expected, the decreasing peak currents are detected with increasing cycle numbers, since the electrode was gradually insulated by the polymer film. After 50 cycles, the response current was lowered down to the baseline indicating that the electrode became totally insulated and thus could not be coated with any further polymer.(11) The PDB film thickness was indicated to be about $27-31 \mathrm{~nm}$ by atomic force microscopy (data not shown) after 50 electropolymerisation cycles, which was in good agreement with previous studies ${ }^{(3,4)}$ of different types of electrodes. To verify the electrochemical performance of the PDB-coated GCE, CV experiments were performed in $5 \mathrm{mM}$ FCA

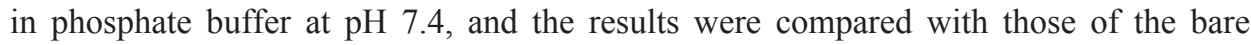
electrode (Fig. 2(b)). In contrast to the CV of the bare electrode, a very small peak current was observed for the PDB-modified GCE, confirming the practically insulated electrode.

\subsection{Sonochemically fabricated microelectrode arrays}

Microelectrode arrays were subsequently formed by sonication of the insulated PDBmodified GCE described in the previous section. The ultrasonic waves have the ability to cause cavitation in the liquid medium as well as acoustic streaming, microstreaming, or micromixing, ${ }^{(12)}$ which resulted in ablation of the ultrathin PDB film. The effects of sonication time (16 to $19 \mathrm{~min}$ ) on microelectrode formation were evaluated electrochemically with a redox couple of FCA (see Fig. 3). A sonication time shorter than 16 min was found to be insufficient to give noticeable voltammetric responses. It is observed that a longer sonication time than 16 min resulted in higher current responses. Peak currents, which indicate planar electrode characteristics, are revealed for 18 and 19 min sonication. In contrast, a sigmoidal voltammetric profile was achieved for the case of 17.30 min sonication, suggesting a true microelectrode-like behavior, which indicated that the electrodes experienced the dominant radial diffusion profile. An inset demonstrates contrasting voltammetric responses of the bare CGE, PDB-coated GCE, and sonochemically fabricated microelectrodes.

Interestingly, the sonication time needed for microelectrode formation in our case $(>16$ min) was much longer than reported previously (5-20 s) for microelectrodes fabricated based on screen-printed or gold-coated glass slide electrodes. ${ }^{(2-6)}$ We postulated that the smooth and glasslike characteristics of the GCE made it more difficult to hole the microarrays compared with screen-printed or gold-coated glass slide electrodes, since 


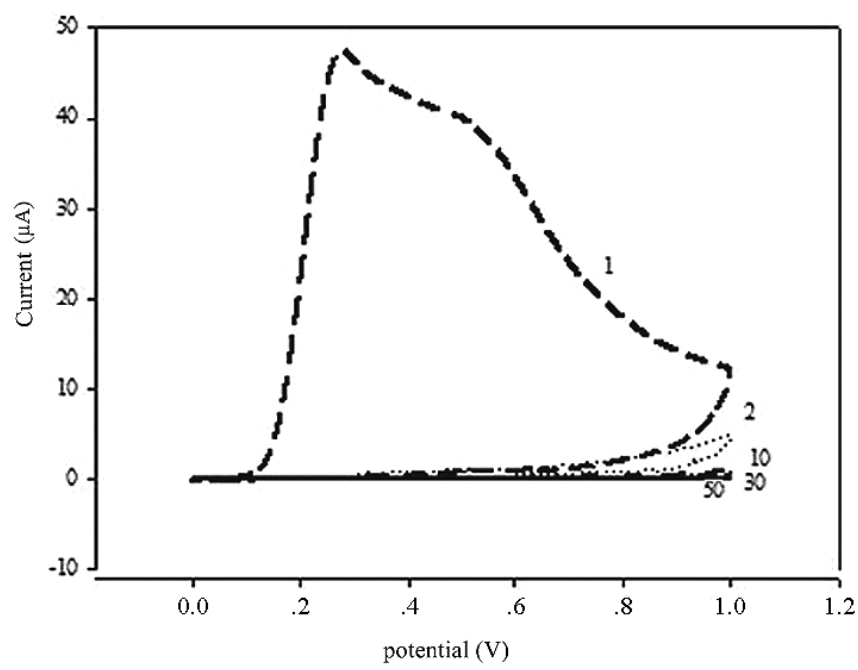

(a)

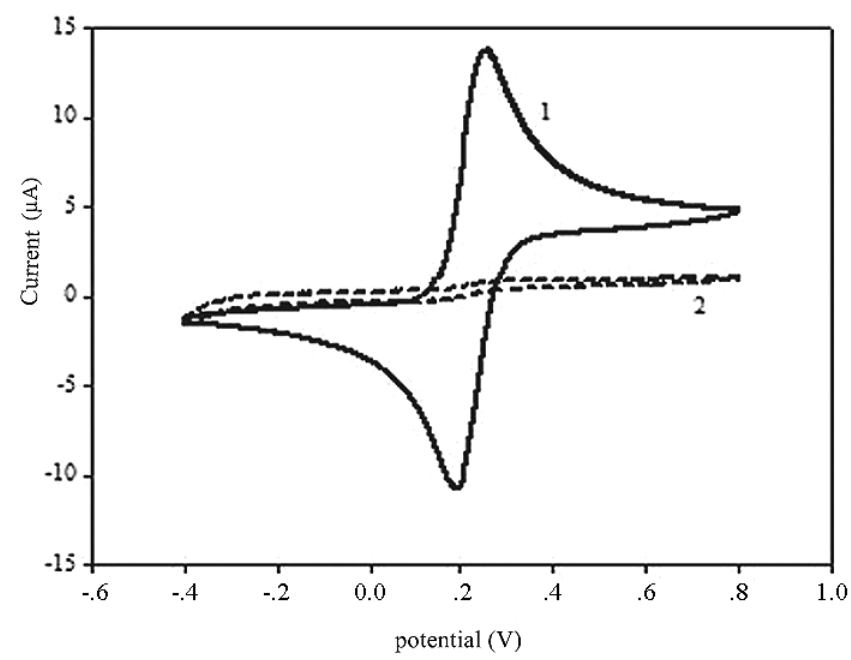

(b)

Fig. 2. Cyclic voltammogram for electropolymerisation (at $20 \mathrm{mVs}^{-1}$ ) at 1, 2, 10, 30, and 50 cycles of $5 \mathrm{mM} \mathrm{1,2} \mathrm{diaminobenzene} \mathrm{dihydrochloride} \mathrm{(a),} \mathrm{and} \mathrm{cyclic} \mathrm{voltammogram} \mathrm{of} \mathrm{bare} \mathrm{(1b)}$ and PDB-coated GCEs in $5 \mathrm{mM}$ FCA buffer (2b).

the acoustic bubbles produced from the ultrasonic frequency could be more easily asymmetrically collapsed on the rough surface. Furthermore, pores on the rough surface may act as the nucleation sites for cavitation on the surface, which can then produce 


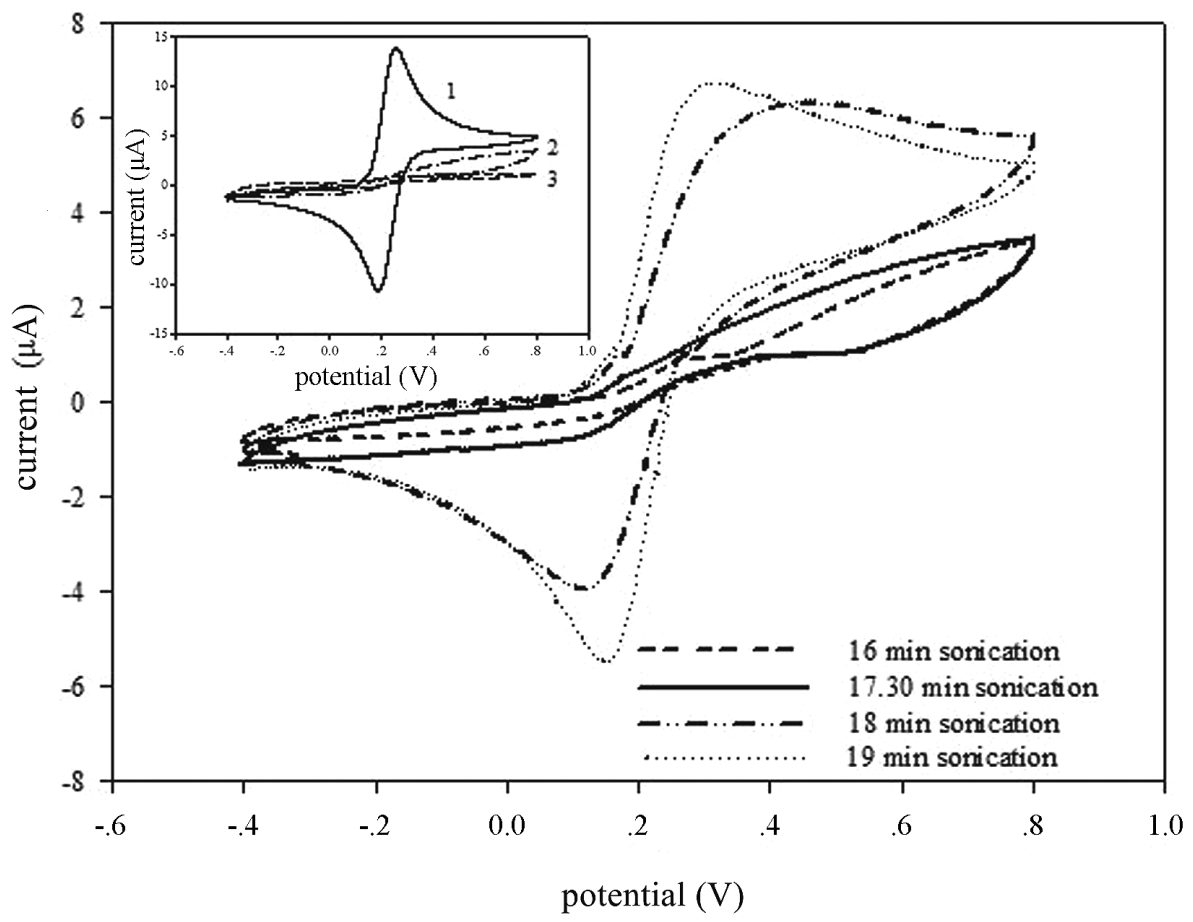

Fig. 3. Cyclic voltammograms of $5 \mathrm{mM} \mathrm{FCA}$ solution at $20 \mathrm{mVs}^{-1}$ under various sonication times. Inset demonstrates CVs of bare GCE (1), $17.30 \mathrm{~min}$ sonochemically ablated PDB-coated GCE (2), and PDB-coated GCE (3).

the larger holes on the ultrathin film. To investigate the electrochemical performance of sonochemically fabricated microelectrode arrays, amperometric responses were tested at different FCA concentrations. The results shown in Fig. 4 reveal a linear correlation between FCA concentrations and current responses at $50 \mathrm{mV}$, suggesting well performance microelectrodes. Figure 5 illustrates SEM images of $17.30 \mathrm{~min}$ sonochemically fabricated microelectrodes under different magnifications. As expected, random microelectrodes were formed owing to the chaotic character of ultrasonic waves. $^{(3)}$ Pores of bimodal sizes of around $2-3 \mu \mathrm{m}$ and submicron (around $0.5 \mu \mathrm{m}$ ) structures were obtained similar to the previous study, ${ }^{(3)}$ with the pore densities estimated at approximately $7 \times 10^{4}$ and $17 \times 10^{4}$ pores $\mathrm{cm}^{-2}$, respectively. Other works reported the population density of $8 \times 10^{4}$ pores $\mathrm{cm}^{-2(4)}$ and $7 \times 10^{4}$ pores $\mathrm{cm}^{-2(3)}$ for PDB-coated gold and screen-printed electrodes, respectively. In contrast to other studies, roundedged pores were not achieved in our case. Instead, sharp-edged geometric forms were detected, which possibly indicated a more brittle PDB film obtained on the glassy carbon surface in comparison with other surfaces. The reproducibility of the fabricated microelectrodes was measured at $3.15 \% \mathrm{RSD}$, and was better than the previously reported value of $6.8 \% \mathrm{RSD}^{(3)}$ obtained using the same sonochemical method. 


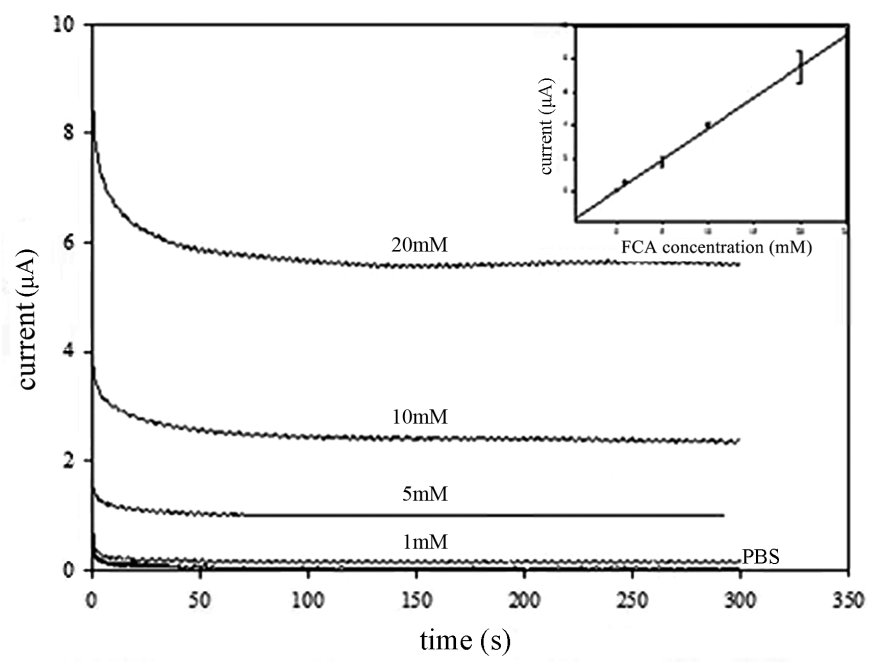

Fig. 4. Amperometric microelectrode array responses to FCA in a concentration range of 1-20 $\mathrm{mM}$. Inset shows an FCA calibration curve.
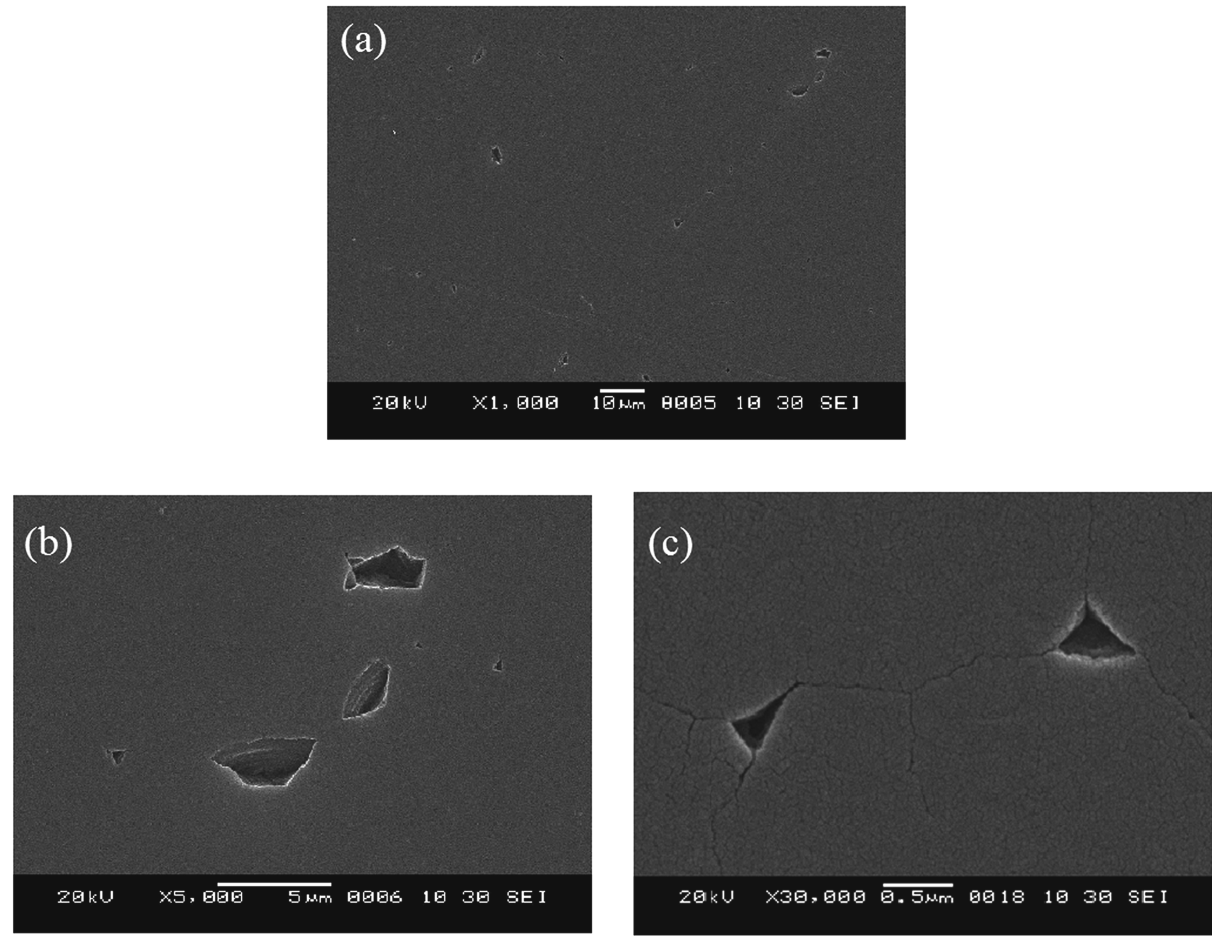

Fig. 5. SEM images of sonochemically ablated PDB-coated GCE after 17.30 min of sonication at (a) $1,000 \times$, (b) $5,000 \times$, and (c) $30,000 \times$. 
To immobilise HRP on microelectrode surfaces, PANI was used as a conducting matrix for enzyme entrapment. HRP and PANI were co-deposited on the sonochemically fabricated microelectrodes described in the previous section. Figure 6 depicts SEM images of HRP/PANI microelectrode arrays under various magnifications. Hemispherical or spherical protrusions of HRP/PANI were observed, however, they were of submicron sizes. Microelectrodes of $\mu \mathrm{m}$-scale structure were found to be recessed, meaning that a longer polymerisation time (or more polymerisation cycles) would be needed if protrusions are required.

For the fabrication of HRP/AuPs/PANI microelectrode arrays, $\mathrm{Au}^{+}$ions were firstly reduced at microelectrode surfaces forming $\mathrm{Au}^{0}$, which then agglomerated into AuPs. Co-deposition of HRP/PANI was subsequently carried out. After $30 \mathrm{~s}$ of electrodeposition, spherical gold particles of around $1 \mu \mathrm{m}$ in diameter were formed on microelectrode surfaces (Fig. 7). However, smaller particles were also observed depending on the sizes of nanocavities. More interestingly, multiparticles were found incorporated into a single pore of $\mu \mathrm{m}$ structure. Moreover, the sigmoidal shape (Fig. 8) was achieved after electrodeposition of AuPs on modified electrodes with the reproducibility of $8.17 \%$ RSD. The increase in surface area of microelectrodes owing
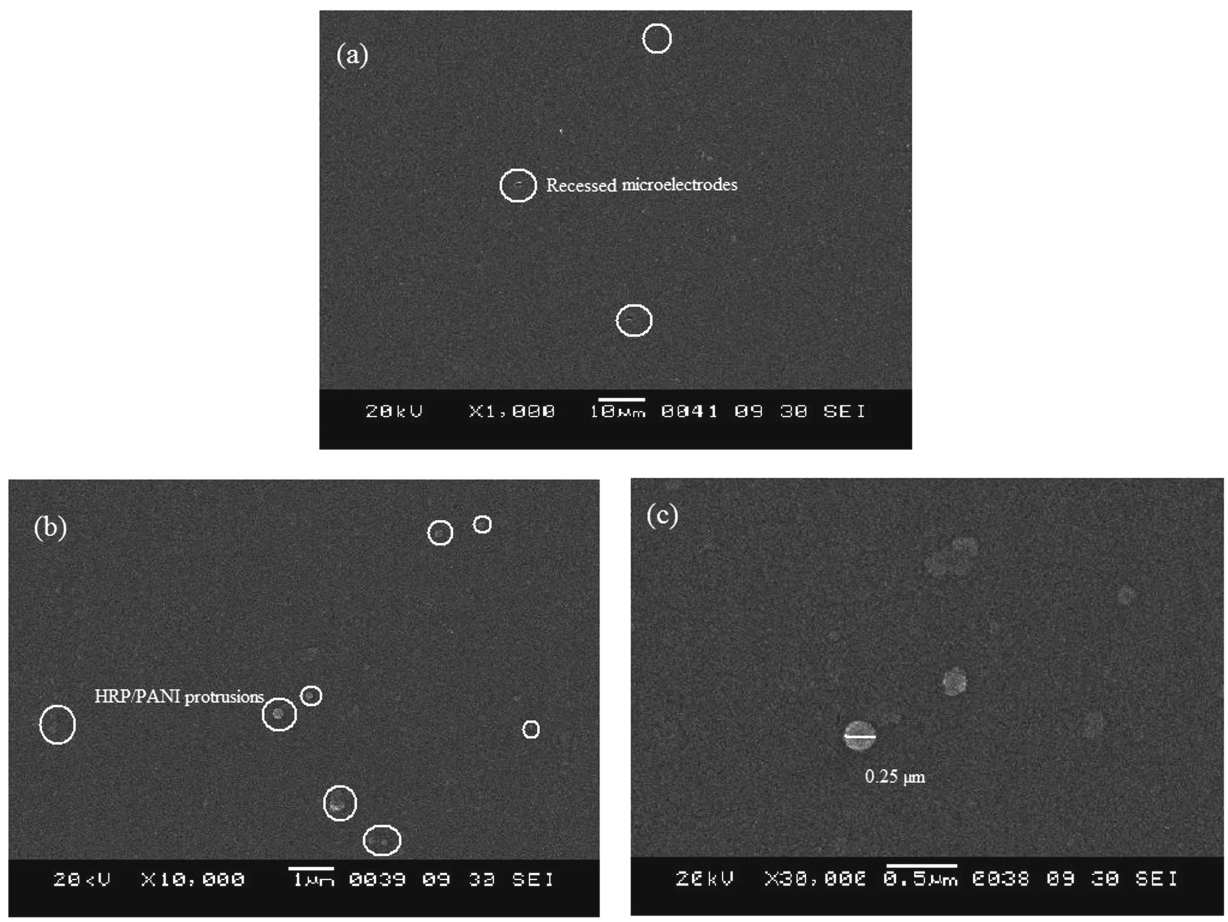

Fig. 6. SEM images of HRP/PANI microelectrode arrays: (a) 1,000×, (b) 10,000×, and (c) 30,000×. 

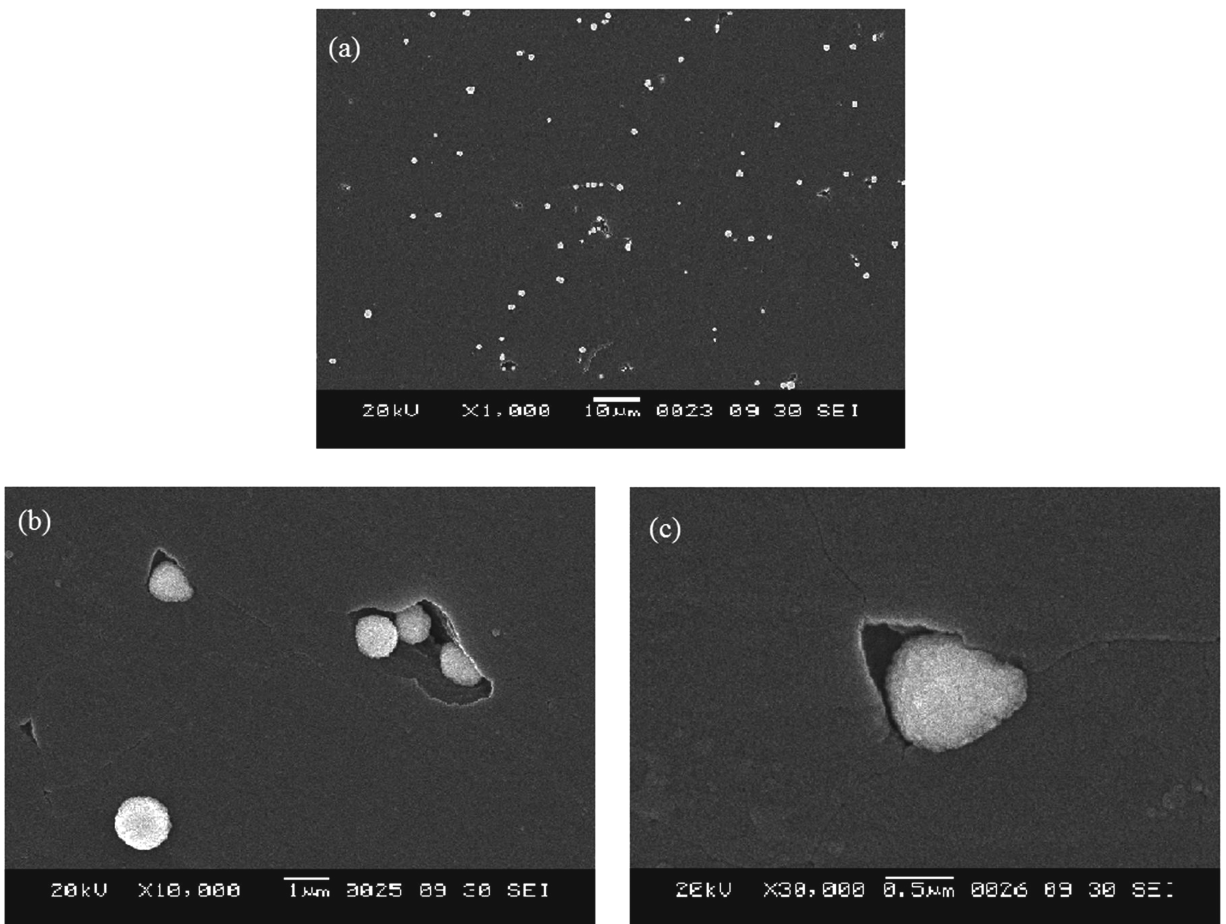

Fig. 7. SEM images of AuPs on microelectrode surfaces: (a) $1,000 \times$, (b) $10,000 \times$, and (c) $30,000 \times$.

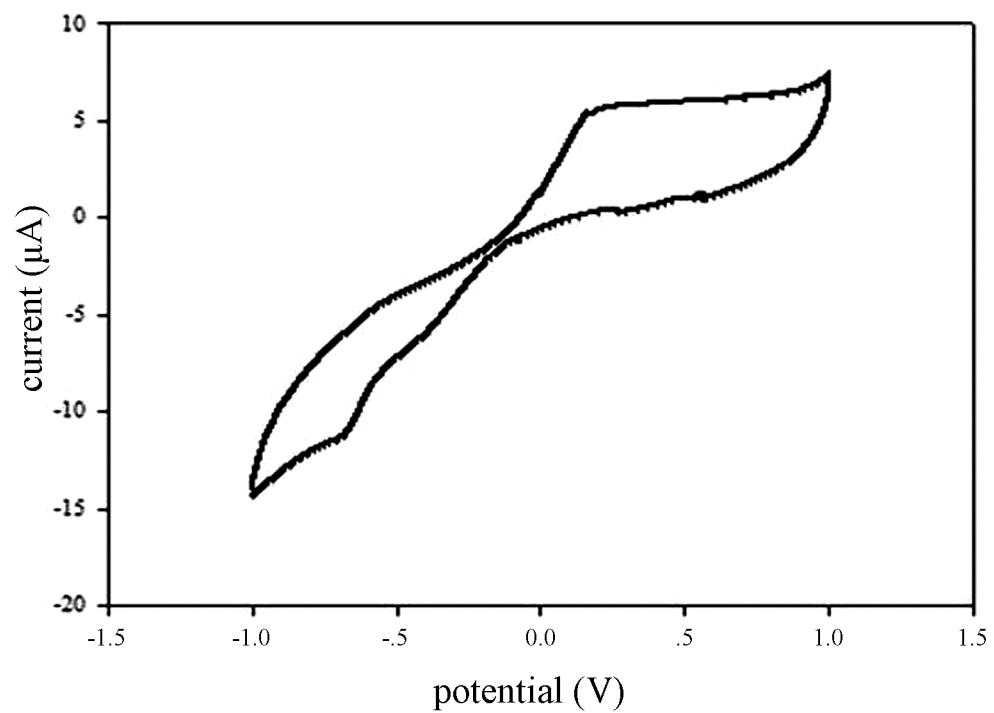

Fig. 8. Cyclic voltammogram of AuP microelectrode arrays (electrodeposition at $-20 \mathrm{mV}$ for $30 \mathrm{~s}$ ) in $5 \mathrm{mM}$ FCA. 
to the deposition of gold particles would thus be advantageous for HRP adsorption and reaction. Upon co-deposition of HRP/PANI onto AuP microelectrodes, the SEM images (Fig. 9) reveal coverage of HRP/PANI film on conductive surfaces that include AuPs and the underlying glassy carbon substrate. The SEM images disclose similar sizes of AuPs and AuPs coated with HRP/PANI, which suggests that an ultrathin HRP/PANI film was electrodeposited on the AuP surfaces. Similar to what was found for HRP/PANI microelectrodes, protrusions were not observed for pores of $\mu \mathrm{m}$-scale structure.

\subsection{Electrochemical performance of $H R P / P A N I$ and AuPs/HRP/PANI microelectrode arrays}

To investigate the electrochemical performance of HRP/PANI and AuPs/HRP/PANI microelectrode arrays, phenol was used as a model analyte in this study, and the reaction mechanisms are as follows: ${ }^{(13)}$

$$
\begin{gathered}
\text { NativeHRP }+\mathrm{H}_{2} \mathrm{O}_{2} \rightarrow \text { Compound I }+\mathrm{H}_{2} \mathrm{O} \\
\text { Compound I }+ \text { phenol }_{\text {red }} \rightarrow{\text { Compound II }+ \text { phenol }_{\mathrm{ox}}}^{\text {Compon }}
\end{gathered}
$$
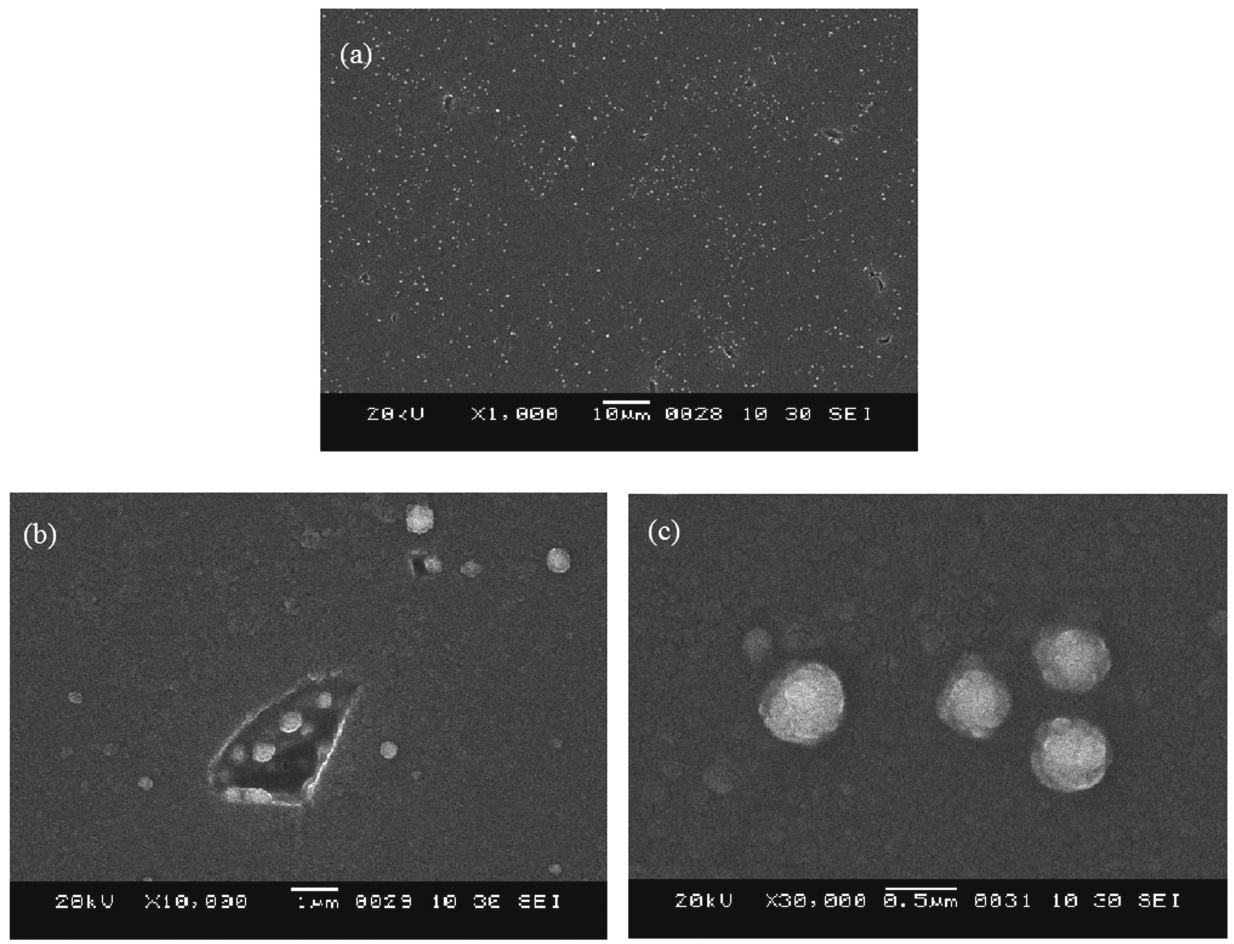

Fig. 9. SEM images of AuPs/HRP/PANI on microelectrode surfaces: (a) 1,000×, (b) 10,000× and (c) $30,000 \times$. 


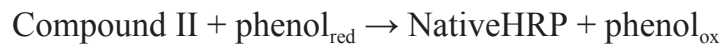

In this case, NativeHRP is first oxidised by $\mathrm{H}_{2} \mathrm{O}_{2}$ to form compound I (eq. (1)), which can then be reduced by phenol resulting in compound II and the oxidised form of phenol (eq. (2)). Finally, NativeHRP is achieved after the reduction of compound II with another molecule of phenol (eq. (3)). Thus, phenol acts as an electron mediator that could be electrochemically reduced back to its initial state at the electrode surface. The reduction current, therefore, is expected to be proportional to the solution phenol concentrations. Moreover, to be certain that the electrochemical responses obtained in our study represent the actual HRP reactions, the experiments were performed for HRP/PANI and HRP-free PANI microelectrodes in a solution of $1 \times 10^{-6} \mathrm{M}$ phenol; the amperometric responses of the enzyme-incorporated microelectrodes showed an 8-fold higher response than those without. This indicated that the electrochemical responses in our study were due mainly to the HRP reaction. Figure 10 demonstrates a schematic diagram of microelectrodes for phenol detection, and Fig. 11 shows amperometric responses of HRP/PANI and

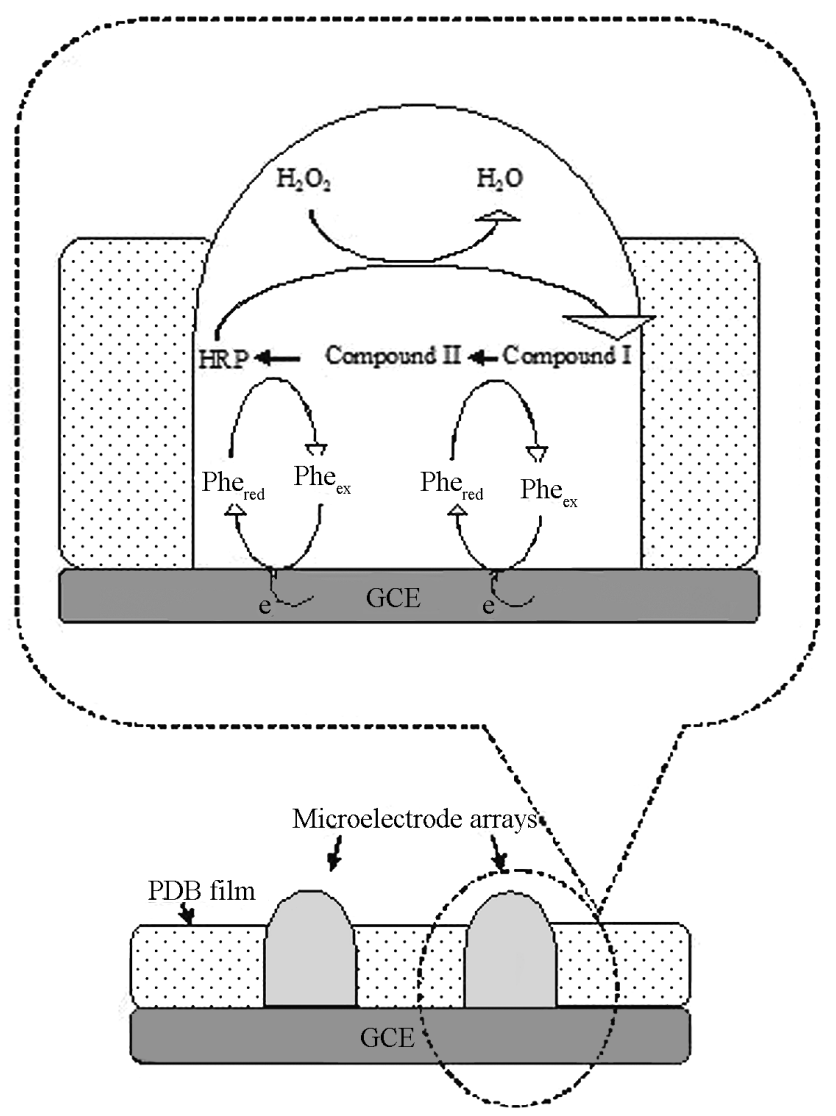

Fig. 10. Schematic diagram of phenol detection in microelectrode arrays. 


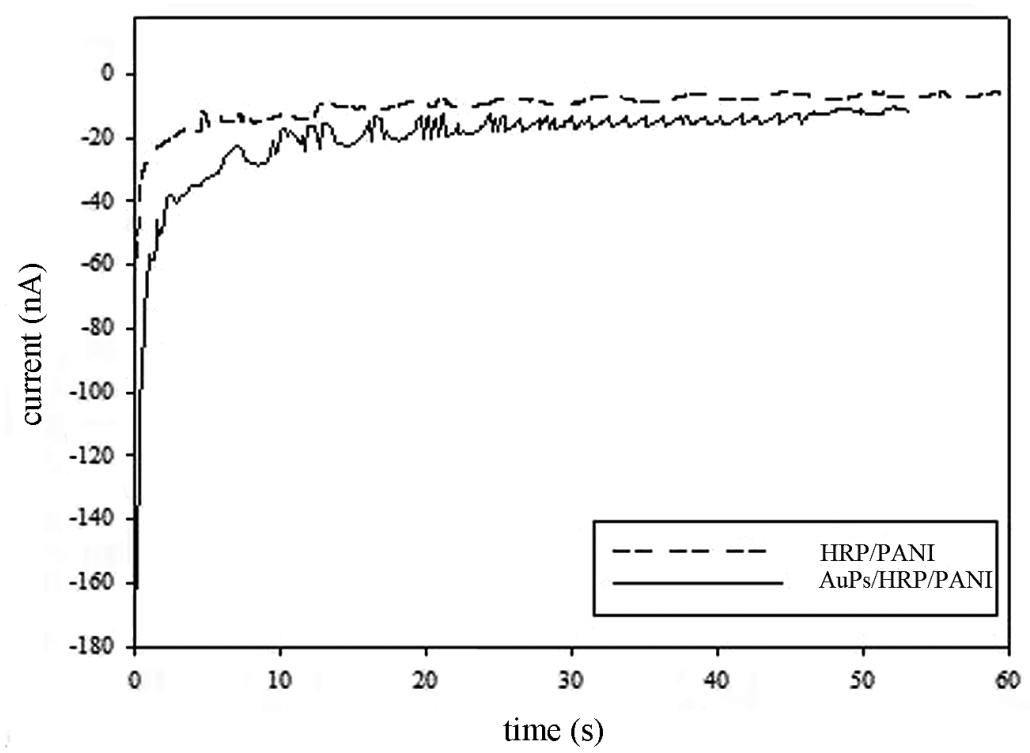

Fig. 11. Comparison of HRP/PANI and AuPs/HRP/PANI microelectrode array responses in $1 \times 10^{-6}$ M phenol solution.

AuPs/HRP/PANI microelectrodes. The electrochemical response of AuPs/HRP/PANI microelectrode arrays in the solution of $1 \times 10^{-6} \mathrm{M}$ phenol at a fixed mole ratio of 0.7 $\mathrm{H}_{2} \mathrm{O}_{2} /$ phenol in acetate buffer $\mathrm{pH} 5.5^{(13,14)}$ was determined to be 2.83 -fold higher $(-22$ $\mathrm{nA}$ vs $-8.87 \mathrm{nA}$ ) than that of HRP/PANI. The results clearly demonstrated significant improvement of electrochemical performance for phenol detection by electrodeposited AuPs on sonochemically fabricated microelectrodes. AuPs apparently increased the interfacial areas for HRP adsorption as well as for catalytic reactions. ${ }^{(9)}$ They also functioned as electron conducting pathways between redox HRP and electrode surfaces ${ }^{(7)}$ and thus favourable results were achieved. Moreover, the amperometric response time of the microelectrodes was achieved in less than $20 \mathrm{~s}$ in comparison to $100 \mathrm{~s}$ using a planar electrode under the same experimental conditions. This is the featured advantage of microelectrode arrays over macroelectrodes.

\section{Conclusions}

Sonochemically fabricated HRP/PANI and AuPs/HRP/PANI microelectrode arrays were constructed in this work for phenol detection. The sonication time needed for microelectrode formation on a PDB-coated GCE in this work was approximately 50 times longer than that in other reports; the smooth glassy carbon surface was probably the underlying reason. The microelectrodes achieved were of bimodal sizes of $\mu \mathrm{m}$ scale and submicron structures with pore densities of approximately $7 \times 10^{4}$ and $17 \times 10^{4}$ 
pores $\mathrm{cm}^{-2}$, respectively. To achieve an HRP microelectrode, PANI was used for enzyme entrapment. Therefore, HRP/PANI and AuPs/HRP/PANI were later electrodeposited on microelectrode surfaces. Spherical AuPs of around $1 \mu \mathrm{m}$ were obtained by the electrodeposition of AuP ions onto microelectrode surfaces. The incorporation of AuPs in an HRP/PANI matrix was postulated to enhance interfacial areas for HRP adsorption as well as function as electron-conducting pathways between the redox HRP and electrode surface. As a consequence, significant improvements of microelectrode performance were achieved. Microelectrode arrays incorporated with AuPs resulted in an approximately 2.83-times-higher response current in comparison to those without particles. Besides that, sensor response time of less than $20 \mathrm{~s}$ was achieved both with and without the incorporation of AuPs. Sonochemically fabricated enzyme/AuP microelectrode arrays showed good potential for biosensor applications.

\section{Acknowledgement}

The authors would like to thank The Office of the Higher Education Commission, Thailand, for the funding under the program Strategic Scholarships for Frontier Research Network for the Joint Ph.D. Program Thai Doctoral degree. Furthermore, this work was also supported by the Higher Education Research Promotion and National Research University Project of Thailand, Office of the Higher Education Commission (FW660I), as well as Chulalongkorn University Centenary Academic Development Project.

\section{References}

1 J. Orozco, C. F.-Sánchez and C. J.-Jorquera: Proc. Chem. 1 (2009) 666.

2 S. Myler, F. Davis, S. D. Collyer and S. P. J. Higson: Biosens. Bioelectron. 20 (2004) 408.

3 A. C. Barton, S. D. Collyer, F. Davis, D. D. Gornall, K. A. Law, E. C. D. Lawrence, D. W. Mills, S. Myler, J. A. Pritchard, M. Thompson and S. P. J. Higson: Biosen. Bioelectron. 20 (2004) 328.

4 S. Myler, S. D. Collyer, F. Davis, D. D. Gornall and S. P. J. Higson: Biosen. Bioelectron. 21 (2005) 666.

5 J. Pritchard, K. Law, A. Vakurov, P. Millner and S. P. J. Higson: Biosen. Bioelectron. 20 (2004) 765.

6 K. A. Law and S. P. J. Higson: Biosen. Bioelectron. 20 (2005) 1914.

7 J. M. Pingarrón, P. Y.-Sedeňo and A. G.-Cortés: Electrochim. Acta 53 (2008) 5848.

8 J. Njagi and S. Andreescu: Biosen. Bioelectron. 23 (2007) 168.

9 H. Yin, S. Ai, S. Shi and L. Zhu: Sens. Actuators, B 137 (2009) 747.

10 T. I. Abdullin, O. V. Bondar, I. I. Nikitina, E. R. Bulatov, M. V. Morozov, A. K. Hilmutdinov, M. K. Salakhov and M. Culha: Bioelectrochem. 77 (2009) 37.

11 S. M. Sayyah, M. M. El-Deeb, S. M. Kamal and R. E. Azooz: J. Appl. Polym. Sci. 112 (2009) 3695.

12 E. Maisonhaute, F. J. Del Campo and R. G. Compton: Ultrason. Sonochem. 9 (2002) 275.

13 S. S. Rosatto, L. T. Kubota and G. Oliveira Neto: Anal. Chim. Acta 390 (1999) 65.

14 L. D. Mello, M. D. P. T. Sotomayor and L. T. Kubota: Sens. Actuators, B 96 (2003) 636. 